PROCEEDINGS OF THE

AMERICAN MATHEMATICAL SOCIETY

Volume 139, Number 11, November 2011, Pages 3831-3843

S 0002-9939(2011)10802-5

Article electronically published on March 16, 2011

\title{
FAMILIES OF DETERMINANTAL SCHEMES
}

\author{
JAN O. KLEPPE AND ROSA M. MIRÓ-ROIG
}

(Communicated by Bernd Ulrich)

\begin{abstract}
Given integers $a_{0} \leq a_{1} \leq \ldots \leq a_{t+c-2}$ and $b_{1} \leq \cdots \leq b_{t}$, we denote by $W(\underline{b} ; \underline{a}) \subset \operatorname{Hilb}^{p}\left(\mathbb{P}^{n}\right)$ the locus of good determinantal schemes $X \subset \mathbb{P}^{n}$ of codimension $c$ defined by the maximal minors of a $t \times(t+c-1)$ homogeneous matrix with entries homogeneous polynomials of degree $a_{j}-b_{i}$. The goal of this paper is to extend and complete the results given by the authors in an earlier paper and determine under weakened numerical assumptions the dimension of $W(\underline{b} ; \underline{a})$ as well as whether the closure of $W(\underline{b} ; \underline{a})$ is a generically smooth irreducible component of $\operatorname{Hilb}^{p}\left(\mathbb{P}^{n}\right)$.
\end{abstract}

\section{INTRODUCTION}

In this paper, we will deal with good and standard determinantal schemes. A scheme $X \subset \mathbb{P}^{n}$ of codimension $c$ is called standard determinantal if its homogeneous saturated ideal can be generated by the maximal minors of a homogeneous $t \times(t+c-1)$ matrix, and $X$ is said to be good determinantal if it is standard determinantal and a generic complete intersection. We denote the Hilbert scheme by $\operatorname{Hilb}^{p}\left(\mathbb{P}^{n}\right)$. Given integers $a_{0} \leq a_{1} \leq \cdots \leq a_{t+c-2}$ and $b_{1} \leq \cdots \leq b_{t}$, we denote by $W(\underline{b} ; \underline{a}) \subset \operatorname{Hilb}^{p}\left(\mathbb{P}^{n}\right)\left(\right.$ resp. $\left.W_{s}(\underline{b} ; \underline{a})\right)$ the locus of good (resp. standard) determinantal schemes $X \subset \mathbb{P}^{n}$ of codimension $c$ defined by the maximal minors of a $t \times(t+c-1)$ homogeneous matrix with entries homogeneous polynomials of degree $a_{j}-b_{i}$.

In [11] and [12, we addressed the following 3 crucial problems:

(1) To determine the dimension of $W(\underline{b} ; \underline{a})$ in terms of $a_{j}$ and $b_{i}$.

(2) Is the closure of $W(b ; a)$ an irreducible component of $\operatorname{Hilb}^{p}\left(\mathbb{P}^{n}\right)$ ?

(3) Is $\operatorname{Hilb}^{p}\left(\mathbb{P}^{n}\right)$ generically smooth along $W(\underline{b} ; \underline{a})$ ?

In 12 we obtained an upper bound for $\operatorname{dim} W(\underline{b} ; \underline{a})$ in terms of $a_{j}$ and $b_{i}$ which was achieved in the cases $2 \leq c \leq 5$ and $n-c>0$ (assuming $\operatorname{char}(k)=0$ if $c=5$ ), and in codimension $c>5$ provided certain numerical conditions are satisfied (see 12, Theorems 3.5 and 4.5; and Corollaries 4.7, 4.10 and 4.14). Concerning problems (2) and (3), we gave in [12] an affirmative answer to both questions in the range $2 \leq c \leq 4$ and $n-c \geq 2$, and in the cases $c \geq 5$ and $n-c \geq 1$ provided certain numerical assumptions are verified (see [12, Corollaries 5.3, 5.7, 5.9 and 5.10; see also [7, [11] for the cases $2 \leq c \leq 3)$. Note that since every element of $W(\underline{b} ; \underline{a})$ has the same Hilbert function, the assumption $n>c$ is close to being necessary

Received by the editors November 10, 2009 and, in revised form, September 17, 2010.

2010 Mathematics Subject Classification. Primary 14M12, 14C05, 14H10, 14J10.

The second author was partially supported by MTM2010-15256. 
for problem (2). Indeed if $n=c$ the problems (2) and (3) become more natural provided we replace $\operatorname{Hilb}^{p}\left(\mathbb{P}^{n}\right)$ by the postulation Hilbert scheme; see [10].

In this work we attempt to extend and complete the results of [11] and [12]. Indeed if $a_{t+3}>a_{t-2}$ we almost solve problem (1) in Theorem [3.2, while Theorem 3.4 and Corollary 3.8, for $c>4$, generalize results of 12 for the problems (2) and (3) substantially. To prove these results we use induction on the codimension by successively deleting the columns of the highest degree and the Eagon-Northcott complex associated to a standard determinantal scheme. We also use the theory of Hilbert flag schemes and the depth of certain mixed determinantal schemes (see Theorem 2.7). We end the paper with two conjectures which are supported by our results and by a huge number of examples computed using Macaulay 2.

Notation. Throughout this paper $\mathbb{P}^{n}$ is the $n$-dimensional projective space over an algebraically closed field $k, R=k\left[x_{0}, x_{1}, \ldots, x_{n}\right]$ and $\mathfrak{m}=\left(x_{0}, \ldots, x_{n}\right)$. By $\mathcal{H} m_{\mathcal{O}_{X}}(\mathcal{F}, \mathcal{G})$ we denote the sheaf of local morphisms between coherent $\mathcal{O}_{X^{-}}$ modules, while $\operatorname{Hom}(\mathcal{F}, \mathcal{G})$ denotes the group of morphisms from $\mathcal{F}$ to $\mathcal{G}$. Moreover we set $\operatorname{hom}(\mathcal{F}, \mathcal{G})=\operatorname{dim}_{k} \operatorname{Hom}(\mathcal{F}, \mathcal{G})$ and we correspondingly use small letters for the dimension, as a $k$-vector space, of similar groups. For any quotient $A$ of $R$ of codimension $c$, we let $K_{A}=\operatorname{Ext}_{R}^{c}(A, R)(-n-1)$.

In the sequel, ${ }_{\mu} \operatorname{Hom}_{R}(M, N)$ denotes homomorphisms of degree $\mu$ of graded $R$-modules. Moreover, we denote the Hilbert scheme by $\operatorname{Hilb}^{p}\left(\mathbb{P}^{n}\right), p$ the Hilbert polynomial, and $(X) \in \operatorname{Hilb}^{p}\left(\mathbb{P}^{n}\right)$ the point which corresponds to the subscheme $X \subset \mathbb{P}^{n}$ with Hilbert polynomial $p$. We denote by $I_{X}$ the saturated homogeneous ideal of $X \subset \mathbb{P}^{n}$. We say that $X$ is general in some irreducible subset $W \subset \operatorname{Hilb}^{p}\left(\mathbb{P}^{n}\right)$ if $(X)$ belongs to a sufficiently small open subset $U$ of $W$ (small enough so that any $(X) \in U$ has all the openness properties that we want to require).

\section{Preliminaries}

This section provides the background and basic results on standard determinantal ideals, good determinantal ideals and mixed determinantal ideals needed in the sequel. We refer to [3, 6] [12] and [15] for the details.

Let $\mathcal{A}=\left(f_{i j}\right)_{i=1, \ldots t}^{j=0, \ldots t+c-2}, \operatorname{deg} f_{i j}=a_{j}-b_{i}$, be a $t \times(t+c-1)$ homogeneous matrix and let

$$
\varphi: F=\bigoplus_{i=1}^{t} R\left(b_{i}\right) \longrightarrow G:=\bigoplus_{j=0}^{t+c-2} R\left(a_{j}\right)
$$

be the graded morphism of free $R$-modules represented by the transpose, $\mathcal{A}^{t}$, of $\mathcal{A}$. Let $I(\mathcal{A})=I_{t}(\mathcal{A})$ be the ideal of $R$ generated by the maximal minors of $\mathcal{A}$. A codimension $c$ subscheme $X \subset \mathbb{P}^{n}$ is said to be standard determinantal if $I_{X}=I(\mathcal{A})$ for some homogeneous $t \times(t+c-1)$ matrix $\mathcal{A}$ as above. Moreover $X$ is good determinantal if $X$ is standard determinantal and a generic complete intersection in $\mathbb{P}^{n}$ ([13], Theorem 3.4). In this paper we suppose $c \geq 2, t \geq 2, b_{1} \leq \ldots \leq b_{t}$ and $a_{0} \leq a_{1} \leq \ldots \leq a_{t+c-2}$. (Note that the case $t=1$ for determinantal schemes corresponds to the well-known complete intersections.)

Let $W(\underline{b} ; \underline{a})$ (resp. $W_{s}(\underline{b} ; \underline{a})$ ) be the stratum in $\operatorname{Hilb}^{p}\left(\mathbb{P}^{n}\right)$ consisting of good (resp. standard) determinantal schemes as above. Since our definition does not assume $\mathcal{A}$ to be minimal (i.e. $f_{i j}=0$ when $b_{i}=a_{j}$ ) for $X=\operatorname{Proj}\left(R / I_{t}(\mathcal{A})\right) \in W(\underline{b} ; \underline{a})$ (or $W_{s}(\underline{b} ; \underline{a})$ ), we must reconsider Corollary 2.6 of $[12$, where $\mathcal{A}$ was supposed minimal 
in the proof (a slight correction to [11 and [12]!). We may, however, use that proof to see that

$W(\underline{b} ; \underline{a}) \neq \emptyset \quad \Leftrightarrow \quad W_{s}(\underline{b} ; \underline{a}) \neq \emptyset \Leftrightarrow a_{i-1} \geq b_{i}$ for all $i$ and $a_{i-1}>b_{i}$ for some $i$.

Indeed if we assume the converse of the condition on the right hand side, then either $I_{t}(\mathcal{A}) \ni 1$ or one of the maximal minors vanishes, i.e. $\operatorname{Proj}\left(R / I_{t}(\mathcal{A})\right) \notin$ $W_{s}(\underline{b} ; \underline{a})$. Conversely assuming the right hand side condition (to simplify notation, assume $a_{i-1} \geq b_{i}$ for $1 \leq i \leq s$ and $a_{i-1}>b_{i}$ for $s<i \leq t$ for some integer $s<t$ ), then we may take $\mathcal{A}=\left(\begin{array}{cc}I & O \\ O & \mathcal{A}^{\prime}\end{array}\right)$, where $I$ is the $s \times s$ identity matrix, $O$ are matrices of zeros and $\mathcal{A}^{\prime}$, for $t-s>1$, the $(t-s) \times(t-s+c-1)$ matrix used in [12], Corollary 2.6 to define a good determinantal scheme (if $t-s=1$ we take the entries of $\mathcal{A}^{\prime}$ to be a regular sequence). We get $\operatorname{Proj}\left(R / I_{t}(\mathcal{A})\right) \in W(\underline{b} ; \underline{a})$ and we easily deduce (2.2). Note that by [12, end of p. 2877 and [12, Remark 3.7, we still have that the closures of $W(\underline{b} ; \underline{a})$ and $W_{s}(\underline{b} ; \underline{a})$ in $\operatorname{Hilb}^{p}\left(\mathbb{P}^{n}\right)$ are equal and irreducible.

Let $A=R / I_{X}$ be the homogeneous coordinate ring of a standard determinantal scheme. By [3], Theorem 2.20 and [6], Corollaries A2.12 and A2.13, the EagonNorthcott complex yields a minimal free resolution of $A$,

$$
\begin{gathered}
0 \longrightarrow \wedge^{t+c-1} G^{*} \otimes S_{c-1}(F) \otimes \wedge^{t} F \longrightarrow \wedge^{t+c-2} G^{*} \otimes S_{c-2}(F) \otimes \wedge^{t} F \longrightarrow \ldots \\
\longrightarrow \wedge^{t} G^{*} \otimes S_{0}(F) \otimes \wedge^{t} F \longrightarrow R \longrightarrow A \longrightarrow 0
\end{gathered}
$$

which allows us to deduce that any standard determinantal scheme is arithmetically Cohen-Macaulay $(\mathrm{ACM})$. Moreover if $M_{\mathcal{A}}:=\operatorname{coker}\left(\varphi^{*}\right)$, then $K_{A}(n+1) \cong$ $S_{c-1} M_{\mathcal{A}}\left(\ell_{c}\right)$, where

$$
\ell_{i}:=\sum_{j=0}^{t+i-2} a_{j}-\sum_{k=1}^{t} b_{k} \text { for } 2 \leq i \leq c .
$$

Let $\mathcal{B}$ be the matrix obtained by deleting the last column of $\mathcal{A}$, let $B=R / I_{B}$ be the $k$-algebra given by the maximal minors of $\mathcal{B}$ and let $M_{\mathcal{B}}$ be the cokernel of $\phi^{*}=\operatorname{Hom}_{R}(\phi, R)$, where $\phi: F=\bigoplus_{i=1}^{t} R\left(b_{i}\right) \rightarrow G^{\prime}:=\bigoplus_{j=0}^{t+c-3} R\left(a_{j}\right)$ is the graded morphism induced by $\mathcal{B}^{t}$. Recall that if $c>2$ there is an exact sequence

$$
0 \longrightarrow B \longrightarrow M_{\mathcal{B}}\left(a_{t+c-2}\right) \longrightarrow M_{\mathcal{A}}\left(a_{t+c-2}\right) \longrightarrow 0
$$

in which $B \longrightarrow M_{\mathcal{B}}\left(a_{t+c-2}\right)$ is a regular section given by the last column of $\mathcal{A}$. Moreover,

$$
0 \longrightarrow M_{\mathcal{B}}\left(a_{t+c-2}\right)^{*}:=\operatorname{Hom}_{B}\left(M_{\mathcal{B}}\left(a_{t+c-2}\right), B\right) \longrightarrow B \longrightarrow A \longrightarrow 0
$$

is exact by [13] or [11] (e.g. see the text after (3.1) of 11]). Note that the proofs of (2.5)-(2.6) rely heavily on the equality $\operatorname{Ann}\left(M_{\mathcal{B}}\right)=I_{B}$ established in [4]. If $c=2\left(\operatorname{codim}_{R} B=1\right)$ we have at least $\operatorname{Ann}\left(M_{\mathcal{B}}\right) I_{t-1}(\mathcal{B}) \subset I_{B} \subset \operatorname{Ann}\left(M_{\mathcal{B}}\right)$ by [4; thus the kernel, $I_{A / B}$, of $B \rightarrow A$ satisfies $I_{A / B}=M_{\mathcal{B}}\left(a_{t+c-2}\right)^{*}\left(\operatorname{resp} .\left.\tilde{I}_{A / B}\right|_{U}=\right.$ $\left.\tilde{M}_{\mathcal{B}}\left(a_{t+c-2}\right)^{*}\right|_{U}$, where $\left.U=Y-V\left(I_{t-1}(\mathcal{B})\right)\right)$ for $c>2($ resp. $c=2)$. Due to the Buchsbaum-Rim resolution of $M:=M_{\mathcal{A}}, M$ is a maximal Cohen-Macaulay $A$-module, and so is $I_{A / B}$ for $c>2$ by (2.6).

By successively deleting columns from the right hand side of $\mathcal{A}$ and taking maximal minors, one gets a flag of standard determinantal subschemes,

$$
\text { (X.) : } X=X_{c} \subset X_{c-1} \subset \ldots \subset X_{2} \subset X_{1} \subset \mathbb{P}^{n},
$$


where each $X_{i+1} \subset X_{i}$ (with ideal sheaf $\mathcal{I}_{X_{i+1} / X_{i}}=\mathcal{I}_{i}$ ) is of codimension $1, X_{i} \subset \mathbb{P}^{n}$ is of codimension $i(i=1, \ldots, c)$ and there exist $\mathcal{O}_{X_{i}}$-modules $\mathcal{M}_{i}$ fitting into short exact sequences

$$
0 \rightarrow \mathcal{O}_{X_{i}}\left(-a_{t+i-1}\right) \rightarrow \mathcal{M}_{i} \rightarrow \mathcal{M}_{i+1} \rightarrow 0 \text { for } 2 \leq i \leq c-1
$$

such that $\mathcal{I}_{i}\left(a_{t+i-1}\right)$ is the $\mathcal{O}_{X_{i}}$-dual of $\mathcal{M}_{i}$ for $2 \leq i \leq c$ (all this holds also for $i=1$ provided we restrict the sheaves to $X_{1}-V\left(I_{t-1}\left(\varphi_{1}\right)\right)$, where $\varphi_{1}$ is given by $X_{1}=$ $\left.V\left(I_{t}\left(\varphi_{1}\right)\right)\right)$. In this context, we let $D_{i}:=R / I_{X_{i}}, I_{D_{i}}:=I_{X_{i}}$ and $I_{i}:=I_{D_{i+1}} / I_{D_{i}}$.

Remark 2.1. Assume $t \geq 2, b_{1} \leq \ldots \leq b_{t}, a_{0} \leq a_{1} \leq \ldots \leq a_{t+c-2}$ and let $\alpha \geq 1$ be an integer. If $X$ is general in $W(\underline{b} ; \underline{a})$ and $a_{i-\min (\alpha, t)}-b_{i} \geq 0$ for $\min (\alpha, t) \leq i \leq t$, then

$$
\operatorname{codim}_{X_{j}} \operatorname{Sing}\left(X_{j}\right) \geq \min \{2 \alpha-1, j+2\} \text { for all } j=2, \cdots, c .
$$

This follows from the theorem of [5], arguing as in [5], Example 2.1. In particular, if $\alpha \geq 3$, we get for each $i>0$ that $X_{i} \hookrightarrow \mathbb{P}^{n}$ and $X_{i+1} \hookrightarrow X_{i}$ are local complete intersections (l.c.i.'s) outside some set $Z_{i}$ of codimension at least $\min (4, i+1)$ in $X_{i+1}$; cf. the paragraph below. Finally note that it is easy to show (2.8) for $(j, \alpha)=(1,2)$ provided $a_{i-2}>b_{i}$ for $2 \leq i \leq t$; cf. [1], (1.10).

Now let $Z \subset X$ (resp. $Z_{i} \subset X_{i}$ ) be some closed subset such that $U:=X-Z \hookrightarrow$ $\mathbb{P}^{n}$ (resp. $\quad U_{i}:=X_{i}-Z_{i} \hookrightarrow \mathbb{P}^{n}$ ) is an l.c.i. By the fact that the first Fitting ideal of $M$ is equal to $I_{t-1}(\varphi)$, we get that $\tilde{M}$ is locally free of rank 1 precisely on $X-V\left(I_{t-1}(\varphi)\right)$ [2, Lemma 1.4.8. Since the set of non-l.c.i points of $X \hookrightarrow \mathbb{P}^{n}$ is precisely $V\left(I_{t-1}(\varphi)\right)$ by e.g. [17, Lemma 1.8, we get that $U \subset X-V\left(I_{t-1}(\varphi)\right)$ and that $\tilde{M}$ is locally free on $U$. Indeed $\mathcal{M}_{i}$ and $\mathcal{I}_{X_{i}} / \mathcal{I}_{X_{i}}^{2}$ are locally free on $U_{i}$ as well as on $U_{i-1} \cap X_{i}$. Note also that since $V\left(I_{t-1}(\mathcal{B})\right) \subset V\left(I_{t}(\mathcal{A})\right)$, we may suppose $Z_{i-1} \subset X_{i}$.

Let us recall the following useful comparison of cohomology groups. If $L$ and $N$ are finitely generated $A$-modules such that $\operatorname{depth}_{I(Z)} L \geq r+1$ and $\tilde{N}$ is locally free on $U:=X-Z$, then the natural map

$$
\operatorname{Ext}_{A}^{i}(N, L) \longrightarrow H_{*}^{i}\left(U, \mathcal{H o m}{ }_{\mathcal{O}_{X}}(\tilde{N}, \tilde{L})\right)
$$

is an isomorphism (resp. an injection) for $i<r$ (resp. $i=r$ ); cf. [9], exp. VI. Note that we interpret $I(Z)$ as $\mathfrak{m}$ if $Z=\emptyset$.

In [12, Conjecture 6.1, we conjectured the dimension of (a non-empty) $W(\underline{b} ; \underline{a})$ in terms of the invariant

$\lambda_{c}:=\sum_{i, j}\left(\begin{array}{c}a_{i}-b_{j}+n \\ n\end{array}\right)+\sum_{i, j}\left(\begin{array}{c}b_{j}-a_{i}+n \\ n\end{array}\right)-\sum_{i, j}\left(\begin{array}{c}a_{i}-a_{j}+n \\ n\end{array}\right)-\sum_{i, j}\left(\begin{array}{c}b_{i}-b_{j}+n \\ n\end{array}\right)+1$.

Here the indices belonging to $a_{j}$ (resp. $b_{i}$ ) range over $0 \leq j \leq t+c-2$ (resp. $1 \leq i \leq t)$ and $\left(\begin{array}{l}a \\ b\end{array}\right)=0$ whenever $a<b$.

Conjecture 2.2. Given integers $a_{0} \leq a_{1} \leq \ldots \leq a_{t+c-2}$ and $b_{1} \leq \ldots \leq b_{t}$, we set $\ell_{i}:=\sum_{j=0}^{t+i-2} a_{j}-\sum_{k=1}^{t} b_{k}$ and $h_{i-3}:=2 a_{t+i-2}-\ell_{i}+n$, for $i=3,4, \ldots, c$. Assume $a_{i-\min ([c / 2]+1, t)} \geq b_{i}$ for $\min ([c / 2]+1, t) \leq i \leq t$. Then we have

$$
\operatorname{dim} W(\underline{b} ; \underline{a})=\lambda_{c}+K_{3}+K_{4}+\ldots+K_{c},
$$


where $K_{3}=\left(\begin{array}{c}h_{0} \\ n\end{array}\right)$ and $K_{4}=\sum_{j=0}^{t+1}\left(\begin{array}{c}h_{1}+a_{j} \\ n\end{array}\right)-\sum_{i=1}^{t}\left(\begin{array}{c}h_{1}+b_{i} \\ n\end{array}\right)$ and in general

$$
K_{i+3}=\sum_{\substack{r+s=i \\
r, s \geq 0}} \sum_{\substack{0 \leq i_{1}<\ldots<i_{r} \leq t+i \\
1 \leq j_{1} \leq \ldots \leq j_{s} \leq t}}(-1)^{i-r}\left(\begin{array}{c}
h_{i}+a_{i_{1}}+\cdots+a_{i_{r}}+b_{j_{1}}+\cdots+b_{j_{s}} \\
n
\end{array}\right)
$$

for $0 \leq i \leq c-3$.

In 12 we proved that the right hand side in the formula for $\operatorname{dim} W(\underline{b} ; \underline{a})$ in Conjecture 2.2 is always an upper bound for $\operatorname{dim} W(\underline{b} ; \underline{a})([12$, Theorem 3.5) and, moreover, that Conjecture 2.2 holds in the range

$$
2 \leq c \leq 5 \text { and } n-c>0 \text { ( supposing } \operatorname{char}(k)=0 \text { if } c=5 \text { ); }
$$

cf. [12, Theorems 3.5 and 4.5, and Corollaries 4.7, 4.10 and 4.14. See also [7, [1] for the cases $2 \leq c \leq 3$. In [10], however, the first author gave a counterexample to Conjecture 2.2 for zero-dimensional schemes (see the section on conjectures in this paper).

Remark 2.3. Conjecture 2.2 holds in codimension $c \geq 6$ provided the numerical conditions of [12, Corollary 4.15 are satisfied. By [12, Remarks 4.16, 4.17 and Corollary 4.18, Conjecture 2.2 holds (without assuming $n>c$ or $\operatorname{char}(k)=0$ ) for any $W(\underline{b} ; \underline{a})$ satisfying

$$
\begin{array}{rrr}
a_{t+3} & >a_{t-1}+a_{t}+a_{t+1}-a_{0}-a_{1} & \text { in codimension } c=5, \\
a_{t+2} & >a_{t-1}+a_{t}-a_{0} & \text { for } n=c=4, \\
a_{t+1} & >a_{t-1} \text { and } a_{i-2}>b_{i} \text { for } 2 \leq i \leq t & \text { for } n=c=3 .
\end{array}
$$

Note that in the case $n=c=3$ we need $a_{i-2}>b_{i}$ (not $a_{i-2} \geq b_{i}$ as assumed in [12]) for $2 \leq i \leq t$ for the proof of [12], Corollary 4.18, to hold; see the last sentence of Remark 2.1.

A goal of this paper is to extend the results summarized in (2.11) and in the above remark. To do this we will need the following result, where $\underline{a}=a_{0}, a_{1}, \ldots, a_{t+c-2}$ and $\underline{a^{\prime}}=a_{0}, a_{1}, \ldots, a_{t+c-3}$.

Proposition 2.4. Let $c \geq 3$, let $(X) \in W(\underline{b} ; \underline{a})$ and suppose $\operatorname{dim} W\left(\underline{b} ; \underline{a^{\prime}}\right) \geq \lambda_{c-1}+$ $K_{3}+K_{4}+\ldots+K_{c-1}$ and $\operatorname{depth}_{I(Z)} B \geq 2$ for a general $Y=\operatorname{Proj}(B) \in W\left(\underline{b} ; \underline{a^{\prime}}\right)$. If

$$
{ }_{0} \operatorname{hom}_{R}\left(I_{Y}, I_{X / Y}\right) \leq \sum_{j=0}^{t+c-3}\left(\begin{array}{c}
a_{j}-a_{t+c-2}+n \\
n
\end{array}\right),
$$

then $\operatorname{dim} W(\underline{b} ; \underline{a})=\lambda_{c}+K_{3}+K_{4}+\ldots+K_{c}$ and (2.12) turns out to be an equality.

Proof. See [10, Proposition 3.4.

Let us recall how we proved Remark 2.3 since we want to generalize that approach. Letting $a=a_{t+i-2}-a_{t+i-1}$ we showed in 12 that $\operatorname{Hom}_{D_{i-1}}\left(I_{i-1}, I_{i}\right) \cong$ $D_{i}(a)$ for $i<c$ using $\operatorname{depth}_{I\left(Z_{i-1}\right)} D_{i} \geq 2$. Indeed this follows from (2.9), i.e. from

$$
H_{*}^{0}\left(U_{i-1}, \mathcal{H o m}\left(\mathcal{I}_{i-1}, \mathcal{I}_{i}\right)\right) \cong H_{*}^{0}\left(U_{i-1}, \mathcal{H o m}_{\mathcal{O}_{X_{i}}}\left(\mathcal{I}_{i-1} \otimes_{\mathcal{O}_{X_{i-1}}} \mathcal{O}_{X_{i}} \otimes \mathcal{I}_{i}^{*}, \mathcal{O}_{X_{i}}\right)\right),
$$

because $\left.\left.\tilde{M}_{i}\right|_{U_{i-1}} \cong \mathcal{I}_{i}^{*}\left(-a_{t+i-1}\right)\right|_{U_{i-1}}$ is locally free, $\left.\left.\tilde{M}_{i}\right|_{U_{i-1}} \cong \tilde{M}_{i-1} \otimes \mathcal{O}_{X_{i}}\right|_{U_{i-1}}$; hence $\left.\left.\mathcal{I}_{i-1} \otimes_{\mathcal{O}_{X_{i-1}}} \mathcal{O}_{X_{i}}\right|_{U_{i-1}} \cong \mathcal{I}_{i}(-a)\right|_{U_{i-1}}$, and note that $U_{i-1} \cap X_{i} \subset U_{i}$. Then 
since

$$
0 \rightarrow \operatorname{Hom}_{R}\left(I_{i-1}, I_{i}\right) \rightarrow \operatorname{Hom}_{R}\left(I_{D_{i}}, I_{i}\right) \rightarrow \operatorname{Hom}_{R}\left(I_{D_{i-1}}, I_{i}\right)
$$

is exact we were able to show (2.12) for $X=X_{c} \subset Y=X_{c-1}$ by putting ${ }_{0} \operatorname{Hom}_{R}\left(I_{D_{c-2}}, I_{c-1}\right)=0$ (through making the minimal generators of $I_{D_{c-2}}$ and $I_{c-1}$ explicit via (2.3) ). Using Proposition 2.4 we obtained Remark 2.3 if the conjecture holds for $W\left(\underline{b} ; \underline{a^{\prime}}\right) \ni Y:=X_{c-1}$.

Let us finish this section by gathering all the results on the depth of mixed determinantal ideals and cogenerated ideals needed in the next section. We start by fixing some notation. Let

$$
\mathcal{A}=\left(\begin{array}{cccc}
f_{1}^{1} & f_{2}^{1} & \cdots & f_{q}^{1} \\
f_{1}^{2} & f_{2}^{2} & \cdots & f_{q}^{2} \\
\vdots & \vdots & \vdots & \vdots \\
f_{1}^{p} & f_{2}^{p} & \cdots & f_{q}^{p}
\end{array}\right)
$$

be a homogeneous $p \times q$ matrix with entries homogeneous polynomials $f_{i}^{j} \in$ $k\left[x_{0}, \cdots, x_{n}\right]$ of degree $a_{j}-b_{i}$. For any choice $(\underline{\alpha} ; \beta)=\left(\alpha_{1}, \cdots, \alpha_{m} ; \beta_{1}, \cdots, \beta_{m}\right)$ of row indexes $1 \leq \alpha_{1}<\cdots<\alpha_{m} \leq p$ and of column indexes $1 \leq \beta_{1}<\cdots<\beta_{m} \leq q$, we denote by $\bar{I}_{(\underline{\alpha} ; \underline{\beta})}(\mathcal{A})$ the ideal cogenerated by $(\underline{\alpha} ; \underline{\beta})$; i.e. $I_{(\underline{\alpha}, \underline{\beta})}$ is the homogeneous ideal of $k\left[x_{0}, \cdots, x_{n}\right]$ generated by all $(m+1) \times(m+1)$ minors of $\mathcal{A}$, all $i \times i$ minors of the rows $1, \cdots, \alpha_{i-1}$ for $i=1, \cdots, m$ and all $i \times i$ minors of the columns $1, \cdots, \beta_{i-1}$ for $i=1, \cdots, m$.

Example 2.5. (1) If $(\underline{\alpha} ; \underline{\beta})=(1, \cdots, t-1 ; 1, \cdots, t-1)$, then $I_{(\underline{\alpha} ; \underline{\beta})}(\mathcal{A})$ is the ideal generated by the $t \times t$ minors of $\mathcal{A}$, i.e., $I_{(\underline{\alpha} ; \underline{\beta})}(\mathcal{A})=I_{t}(\mathcal{A})$.

(2) Let $\mathcal{A}$ be a homogeneous $p \times q$ matrix and let $\mathcal{A}_{i}$ be the matrix obtained by deleting the last $i-1$ columns of $\mathcal{A}$. We assume $p \leq q$ and we fix an integer $m<p$ and $(\underline{\alpha} ; \underline{\beta})=\left(1, \cdots, m ; \beta_{1}, \cdots, \beta_{m}\right)$. Set $j:=\min \left\{i \mid \beta_{i}>i\right\}, c_{1}=1$ and $c_{s}=q+2-\beta_{m+2-s}$ for $2 \leq s \leq m+2-j$. The ideal cogenerated by $(\underline{\alpha} ; \beta)=$ $\left(1, \cdots, m ; \beta_{1}, \cdots, \beta_{m}\right)$ can be identified with the following mixed determinantal ideal

$$
I_{(\alpha ; \beta)}(\mathcal{A})=I_{m+1}\left(\mathcal{A}_{c_{1}}\right)+I_{m}\left(\mathcal{A}_{c_{2}}\right)+\cdots+I_{j}\left(\mathcal{A}_{c_{m+2-j}}\right),
$$

where $I_{\lambda}\left(\mathcal{A}_{\varrho}\right)$ denotes the ideal generated by all $\lambda \times \lambda$ minors of $\mathcal{A}_{\varrho}$.

Theorem 2.6. Let $\mathcal{A}=\left(x_{i, j}\right)$ be a $p \times q$ matrix of indeterminates and let $(\underline{\alpha} ; \underline{\beta})=$ $\left(\alpha_{1}, \cdots, \alpha_{m} ; \beta_{1}, \cdots, \beta_{m}\right)$ be a choice of $m$ row indexes and of $m$ column indexes. Let $I_{(\underline{\alpha} ; \underline{\beta})}$ be the ideal cogenerated by $(\underline{\alpha} ; \underline{\beta})$. Then, $I_{(\underline{\alpha} ; \underline{\beta})}(\mathcal{A})$ is a Cohen-Macaulay ideal and

$$
h t\left(I_{(\underline{\alpha} ; \underline{\beta})}(\mathcal{A})\right)=p q-(p+q+1) m+\sum_{i=1}^{m}\left(\alpha_{i}+\beta_{i}\right) .
$$

Proof. See [3], Corollary 5.12.

We are now ready to state the main result of this section and to prove that the above formula for the height of a cogenerated ideal associated to a matrix with entries that are indeterminates also works for a general homogeneous matrix with entries homogeneous polynomials of positive degree. Indeed, we have 
Theorem 2.7. Fix integers $b_{1}, \cdots, b_{q}$ and $a_{1}, \cdots, a_{p}$. Let $\mathcal{A}=\left(f_{i}^{j}\right)_{i=1, \cdots, q}^{j=1, \cdots, p}$ be a general homogeneous $p \times q$ matrix $\mathcal{A}$ with entries that are homogeneous forms of degree $a_{j}-b_{i}$. Assume that $a_{j}>b_{i}$ for all $j, i$. Then, for any choice $(\underline{\alpha} ; \underline{\beta})=$ $\left(\alpha_{1}, \cdots, \alpha_{m} ; \beta_{1}, \cdots, \beta_{m}\right)$ of $m$ row indexes and of $m$ column indexes, we have that $I_{(\underline{\alpha} ; \underline{\beta})}(\mathcal{A})$ is a Cohen-Macaulay ideal and

$$
h t\left(I_{(\underline{\alpha} ; \underline{\beta})}(\mathcal{A})\right)=p q-(p+q+1) m+\sum_{i=1}^{m}\left(\alpha_{i}+\beta_{i}\right)
$$

provided $p q-(p+q+1) m+\sum_{i=1}^{m}\left(\alpha_{i}+\beta_{i}\right) \leq n+1$.

Proof. We clearly have $h t\left(I_{(\underline{\alpha} ; \underline{\beta})}(\mathcal{A})\right) \leq p q-(p+q+1) m+\sum_{i=1}^{m}\left(\alpha_{i}+\beta_{i}\right)$, and it will be enough to construct an example of a homogeneous $p \times q$ matrix $\mathcal{M}$ with entries homogeneous forms $f_{i}^{j} \in k\left[x_{0}, \cdots, x_{n}\right]$ such that the ideal $I_{(\underline{\alpha} ; \underline{\beta})}(\mathcal{M})$ cogenerated by $(\underline{\alpha} ; \underline{\beta})$ is Cohen-Macaulay and $h t\left(I_{(\underline{\alpha} ; \beta)}(\mathcal{M})\right)=p q-(p+q+1) m+\sum_{i=1}^{m}\left(\alpha_{i}+\beta_{i}\right)$. Therefore, let $n+1 \geq p q-(p+q+1) m+\sum_{i=1}^{m}\left(\alpha_{i}+\beta_{i}\right)$. We will distinguish 2 cases:

Case 1. Assume $a_{j}-b_{i}=1$ for all $i, j$ (i.e. the entries of the matrix $\mathcal{A}$ are linear forms). By Theorem 2.6 for any choice of $p, q$ and $(\underline{\alpha} ; \underline{\beta})$, we have the matrix $\overline{\mathcal{A}}=\left(x_{i, j}\right)$ of indeterminates and the ideal $I_{(\underline{\alpha} ; \underline{\beta})}(\overline{\mathcal{A}}) \subset S:=k\left[x_{1,1} \cdots, x_{p, q}\right]$ cogenerated by $(\underline{\alpha} ; \underline{\beta})$ is Cohen-Macaulay of height

$$
h t\left(I_{(\underline{\alpha} ; \underline{\beta})}(\overline{\mathcal{A}})\right)=p q-(p+q+1) m+\sum_{i=1}^{m}\left(\alpha_{i}+\beta_{i}\right) .
$$

We choose $p q-n-1$ general linear forms $\ell_{1}, \cdots, \ell_{p q-n-1} \in S=k\left[x_{1,1} \cdots, x_{p, q}\right]$ and we set $S /\left(\ell_{1}, \cdots, \ell_{p q-n-1}\right) \cong k\left[x_{0}, x_{1}, \cdots, x_{n}\right]=: R$. Let us call $I \subset R$ the ideal of $R$ isomorphic to the ideal $I_{(\underline{\alpha} ; \beta)}(\overline{\mathcal{A}}) /\left(\ell_{1}, \cdots, \ell_{p q-n-1}\right)$ of $S /\left(\ell_{1}, \cdots, \ell_{p q-n-1}\right)$. Note that $I$ is a Cohen-Macaulay ideal of height $h t(I)=p q-(p+q+1) m+\sum_{i=1}^{m}\left(\alpha_{i}+\beta_{i}\right)$ and, in addition, $I$ is nothing but the ideal $I_{(\underline{\alpha} ; \underline{\beta})}(\mathcal{M})$, where $\mathcal{M}=\left(m_{i}^{j}\right)$ is a $p \times q$ homogeneous matrix with entries linear forms in $k\left[x_{0}, x_{1}, \cdots, x_{n}\right]$ obtained from $\overline{\mathcal{A}}=\left(x_{i, j}\right)$ by substituting using the equations $\ell_{1}, \cdots, \ell_{p q-n-1}$, which proves what we want.

Case 2. Assume $a_{j}-b_{i} \geq 1$ for all $i, j$. In this case, it is enough to raise the entry $m_{i}^{j}$ of the above matrix $\mathcal{M}$ to the power $a_{j}-b_{i}$.

Remark 2.8. Fix integers $a_{0} \leq a_{1} \leq \ldots \leq a_{t+c-2}$ and $b_{1} \leq \ldots \leq b_{t}$. Let $X \subset \mathbb{P}^{n}$, $(X) \in W(\underline{b} ; \underline{a})$ be a general determinantal ideal associated to a $t \times(t+c-1)$ matrix $\mathcal{A}$ represented by a graded morphism as in (2.1). Let

$$
\text { (X.) : } X=X_{c} \subset X_{c-1} \subset \ldots \subset X_{2} \subset X_{1} \subset \mathbb{P}^{n}
$$

be the flag of standard determinantal subschemes that we obtain by successively deleting columns from the right hand side of $\mathcal{A}$ and let $\varphi_{i}$ be the graded morphism associated to the matrix which defines $X_{i}$. Assume $a_{0}>b_{t}$ and $c \geq 3$. Applying Theorem 2.7, we get the following formula:

$$
\operatorname{dim} R /\left(I_{t-1}\left(\varphi_{1}\right)+I_{t}\left(\varphi_{c-1}\right)\right)=\operatorname{dim} D_{c-1}-2 .
$$


Even more, if $\operatorname{dim} D_{c-1} \geq 3$ and $c \geq 4$, we have the equalities

$$
\operatorname{dim} R /\left(I_{t-1}\left(\varphi_{i}\right)+I_{t}\left(\varphi_{c-1}\right)\right)=\operatorname{dim} D_{c-1}-i-1
$$

for $1 \leq i \leq 2$, which will play an important role in the next section.

Remark 2.9. If $t=2$ we can prove (2.16) directly as follows. Let $\mathcal{A}=[\mathcal{C}, \mathcal{D}, v]$, where $\mathcal{C}$ is a 2 by 2 general enough matrix in the variables $x_{0}, x_{1}, x_{2}, x_{3}$ (e.g. with rows $\left(x_{0}^{a_{0}-b_{1}}, x_{1}^{a_{1}-b_{1}}\right)$ and $\left.\left(x_{2}^{a_{0}-b_{2}}, x_{3}^{a_{1}-b_{2}}\right)\right), v$ is some column and $\mathcal{D}$ is a 2 by $c-2$ matrix whose first row is $\left(x_{4}^{a_{2}-b_{1}}, x_{5}^{a_{3}-b_{1}}, \ldots, x_{c}^{a_{c-2}-b_{1}}, 0\right)$ and whose second row is $\left(0, x_{4}^{a_{3}-b_{2}}, x_{5}^{a_{4}-b_{2}}, \ldots, x_{c}^{a_{c-1}-b_{2}}\right)$. Note that $\varphi_{1}$ corresponds to $\mathcal{C}$ and $\varphi_{c-1}$ to $[\mathcal{C}, \mathcal{D}]$. Since $\mathcal{C}$ is general, we get $\operatorname{codim}_{R} R / I_{t-1}\left(\varphi_{1}\right)=4$; i.e. the radical of $I_{t-1}\left(\varphi_{1}\right)$ is $\left(x_{0}, x_{1}, x_{2}, x_{3}\right)$. Since it is clear that the radical of the ideal generated by the maximal minors of $\mathcal{D}$ is $\left(x_{4}, x_{5}, \ldots, x_{c}\right)$, it follows that the scheme $X_{c-1}=$ $\operatorname{Proj}\left(D_{c-1}\right) \in W\left(\underline{b} ; \underline{a^{\prime}}\right)$ given by the maximal minors of $[\mathcal{C}, \mathcal{D}]$ satisfies $(2.16)$. The general element $X$ of $W(\underline{b} ; \underline{a})$ will therefore have a flag where $X_{c-1}$ satisfies (2.16).

\section{Smoothness And Dimension of the Determinantal LOCUS}

This section is the heart of the paper and contains the results which generalize quite a lot of our previous contributions to problems (1)-(3) stated in the introduction.

Proposition 3.1. With notation as in Remark 2.8, let $c \geq 3$ and suppose (2.16) (this holds if $\left.a_{0}>b_{t}\right)$. If $a_{t+c-2}>a_{t-2}$, then (2.12) holds for $X:=X_{c} \subset Y:=$ $X_{c-1}$, i.e.

$$
{ }_{0} \operatorname{hom}_{R}\left(I_{D_{c-1}}, I_{D_{c} / D_{c-1}}\right) \leq \sum_{j=0}^{t+c-3}\left(\begin{array}{c}
a_{j}-a_{t+c-2}+n \\
n
\end{array}\right) .
$$

In particular we get $\operatorname{dim} W(\underline{b} ; \underline{a})=\lambda_{c}+K_{3}+K_{4}+\ldots+K_{c}$ provided $\operatorname{dim} W\left(\underline{b} ; \underline{a^{\prime}}\right)=$ $\lambda_{c-1}+K_{3}+K_{4}+\ldots+K_{c-1}$, where $\underline{a^{\prime}}=a_{0}, a_{1}, \ldots, a_{t+c-3}$.

Proof. We claim that $\operatorname{depth}_{I\left(Z_{j}\right)} D_{c-1} \geq 2$ for all $j$ satisfying $0<j<c-1$. Indeed by the discussion right after Remark 2.1 we see that we may take $I\left(Z_{j}\right)$ to be the ideal $I_{t-1}\left(\varphi_{j}\right) \subset R$ of submaximal minors of the matrix which defines $X_{j}$, and $I\left(Z_{j}\right) D_{c-1}$ to be $\left(I_{t-1}\left(\varphi_{j}\right)+I_{t}\left(\varphi_{c-1}\right)\right) D_{c-1}$. It follows that $\operatorname{depth}_{I_{t-1}\left(\varphi_{j}\right)} D_{c-1} \geq 2$ for $j=1$, i.e. $\operatorname{dim} D_{c-1}-\operatorname{dim} D_{c-1} /\left(I_{t-1}\left(\varphi_{1}\right)+I_{t}\left(\varphi_{c-1}\right)\right) D_{c-1} \geq 2$, implies the claim. Hence we conclude the proof of the claim by (2.16).

For every $j, 0<j<c-1$, put $a:=a_{t+j-1}-a_{t+c-2}$. We claim that

$$
\operatorname{Hom}_{D_{j}}\left(I_{j}, I_{c-1}\right) \cong D_{c-1}(a) \text {. }
$$

To prove this claim we remark that $\operatorname{depth}_{I\left(Z_{j}\right)} I_{c-1} \geq 2$ since $I_{c-1}$ is maximally CM. Using that $\mathcal{I}_{j}$ is locally free on $U_{j}$ and the arguments in the text before (2.13) (see the text accompanying (2.7) for $j=1$ ), we get

$$
\left.\left.\left.\mathcal{I}_{j} \otimes \mathcal{O}_{X_{j}} \mathcal{O}_{X_{c-1}}\right|_{U_{j}} \cong \mathcal{I}_{j} \otimes_{\mathcal{O}_{X_{j}}} \mathcal{O}_{X_{j+1}} \otimes \ldots \otimes_{\mathcal{O}_{X_{c-2}}} \mathcal{O}_{X_{c-1}}\right|_{U_{j}} \cong \mathcal{I}_{c-1}(-a)\right|_{U_{j}} .
$$

It follows that $\mathcal{H o m}_{\mathcal{O}_{X_{j}}}\left(\mathcal{I}_{j}, \mathcal{I}_{c-1}\right)(-a) \cong \mathcal{H o m}_{\mathcal{O}_{X_{c-1}}}\left(\mathcal{I}_{c-1}, \mathcal{I}_{c-1}\right) \cong \mathcal{O}_{X_{c-1}}$ are isomorphic as sheaves on $U_{j} \cap X_{c-1}$; i.e. we get that $H_{*}^{0}\left(U_{j}, \mathcal{H o m}\left(\mathcal{I}_{j}, \mathcal{I}_{c-1}\right)\right) \cong$ $H_{*}^{0}\left(U_{j}, \mathcal{O}_{X_{c-1}}\right)(a)$ and hence the claim from $\operatorname{depth}_{I\left(Z_{j}\right)} D_{c-1}=\operatorname{depth}_{I\left(Z_{j}\right)} I_{c-1} \geq 2$ and (2.9). 
$(3.1)$

Now we repeatedly use the exact sequence

$$
0 \rightarrow D_{c-1}(a) \cong \operatorname{Hom}_{D_{j}}\left(I_{j}, I_{c-1}\right) \rightarrow \operatorname{Hom}_{R}\left(I_{D_{j+1}}, I_{c-1}\right) \rightarrow \operatorname{Hom}_{R}\left(I_{D_{j}}, I_{c-1}\right) \rightarrow
$$

for $j=c-2, c-3, \ldots, 1$. Since $a_{t+j-1} \leq a_{t+c-2}$, we have $\operatorname{dim} D_{c-1}(a)_{0}=\left(\begin{array}{c}a+n \\ n\end{array}\right)$. It follows that

$$
{ }_{0} \operatorname{hom}\left(I_{D_{c-1}}, I_{c-1}\right) \leq{ }_{0} \operatorname{hom}\left(I_{D_{1}}, I_{c-1}\right)+\sum_{i=t}^{t+c-3}\left(\begin{array}{c}
a_{i}-a_{t+c-2}+n \\
n
\end{array}\right),
$$

where we have replaced $a_{t+j-i}$ by $a_{i}$, in which case $1 \leq j \leq c-2$ corresponds to $t \leq i \leq t+c-3$.

It remains to prove that ${ }_{0} \operatorname{hom}\left(I_{D_{1}}, I_{c-1}\right) \leq \sum_{i=0}^{t-1}\left(\begin{array}{c}a_{i}-a_{t+c-2}+n \\ n\end{array}\right)$ since we then by Proposition 2.4 get the dimension formula. Using that $X_{1}=\operatorname{Proj}\left(D_{1}\right)$ is a hypersurface of degree $\ell_{1}$, we find ${ }_{0} \operatorname{hom}\left(I_{D_{1}}, I_{c-1}\right) \cong \operatorname{dim} I_{c-1}\left(\ell_{1}\right)_{0}$, where $\ell_{k}=\sum_{j=0}^{t+k-2} a_{j}-\sum_{i=1}^{t} b_{i}$. Now we have to make the degrees of the minimal generators of $I_{c-1}$ explicit. Taking a close look at (2.3), we see that a minimal generator $f$ of $I_{c-1} \cong I_{D_{c}} / I_{D_{c-1}}$ of the smallest possible degree has degree $s\left(I_{c-1}\right):=\ell_{c}-\sum_{j=t-1}^{t+c-3} a_{j}$ because $a_{0} \leq a_{1} \leq \cdots \leq a_{t+c-2}$. Since $\ell_{1}-s\left(I_{c-1}\right)=$ $a_{t-1}-a_{t+c-2} \leq 0$ by the definition of $\ell_{k}$, we get either $\operatorname{dim} I_{c-1}\left(\ell_{1}\right)_{0}=0$ or $a_{t-1}=a_{t+c-2}$. In the latter case the assumption $a_{t+c-2}>a_{t-2}$ implies that the degrees of all minimal generators, except for $f$, are strictly greater than $s\left(I_{c-1}\right)$; i.e. we get $\operatorname{dim} I_{c-1}\left(\ell_{1}\right)_{0}=\left(\begin{array}{c}a_{t-1}-a_{t+c-2}+n \\ n\end{array}\right)$ and we are done.

By repeatedly using Proposition 3.1 we get

Theorem 3.2. Let $X \subset \mathbb{P}^{n},(X) \in W(\underline{b} ; \underline{a})$, be a general determinantal scheme and suppose $a_{0}>b_{t}$. Moreover if $c \geq 6$ we suppose $a_{t+3}>a_{t-2}$ (or $a_{t+4}>a_{t-2}$ provided chark $=0$ ), and if $3 \leq c \leq 5$ we suppose $a_{t+c-2}>a_{t-2}$. Then we have

$$
\operatorname{dim} W(\underline{b} ; \underline{a})=\lambda_{c}+K_{3}+K_{4}+\cdots+K_{c} .
$$

Proof. If $3 \leq c \leq 6$ (chark $=0$ if $c=6$ ) and $a_{t+c-2}>a_{t-2}$ we use (2.11) to find $\operatorname{dim} W\left(\underline{b} ; \underline{a^{\prime}}\right)$ and we conclude the proof by Proposition 3.1 .

If $c \geq 6$ and $a_{t+3}>a_{t-2}$ (resp. $a_{t+4}>a_{t-2}$ if $c \geq 7$ ) we repeatedly use Proposition 3.1 to reduce to the case $c=5$ (resp. $c=6$ ), and we conclude by the first part of the proof (note that the assumption $a_{t+c-2}>a_{t-2}$ of Proposition 3.1 is satisfied in this induction).

Remark 3.3. We expect that the assumption $a_{0}>b_{t}$ can be weakened in Theorem 3.2. as well as in (2.16). At least it does for $c=3$ provided we assume $a_{i-2}>b_{i}$ for $2 \leq i \leq t$. Indeed since $I_{t}\left(\varphi_{2}\right) \subset I_{t-1}\left(\varphi_{1}\right)$ we first show (2.16) using Remark 2.1. Then the proof above applies to conclude as in Theorem 3.2 provided $a_{t+1}>a_{t-2}$; cf. Remark 2.3.

If the condition (2.17) is satisfied, then we can prove the following result for $\overline{W(\underline{b} ; \underline{a})}$ to be a generically smooth irreducible component.

Theorem 3.4. Let $X \subset \mathbb{P}^{n},(X) \in W(\underline{b} ; \underline{a})$, be a general determinantal scheme of dimension $n-c \geq 1$, let $c>2$ and let $X=X_{c} \subset X_{c-1} \subset \ldots \subset X_{2} \subset \mathbb{P}^{n}$, $X_{i}=\operatorname{Proj}\left(D_{i}\right)$, be the flag obtained by successively deleting columns from the right hand side. If $a_{0}>b_{t}$,

$$
{ }_{0} \operatorname{Ext}_{D_{2}}^{1}\left(I_{D_{2}} / I_{D_{2}}^{2}, I_{2}\right)=0 \text { and }{ }_{0} \operatorname{Ext}_{D_{3}}^{1}\left(I_{D_{3}} / I_{D_{3}}^{2}, I_{i}\right)=0 \text { for } i=3, \ldots, c-1,
$$


then $\overline{W(\underline{b} ; \underline{a})}$ is a generically smooth irreducible component of the Hilbert scheme $\operatorname{Hilb}^{p}\left(\mathbb{P}^{n}\right)$.

Remark 3.5. If $n-c \geq 2$, then we have ${ }_{0} \operatorname{Ext}_{D_{2}}^{1}\left(I_{D_{2}} / I_{D_{2}}^{2}, I_{2}\right)=0$ provided $c=3$, and ${ }_{0} \operatorname{Ext}_{D_{2}}^{1}\left(I_{D_{2}} / I_{D_{2}}^{2}, I_{2}\right)={ }_{0} \operatorname{Ext}_{D_{3}}^{1}\left(I_{D_{3}} / I_{D_{3}}^{2}, I_{3}\right)=0$ provided $c=4$ by [12], (5.4)(5.8). Hence the conclusion of Theorem 3.4 holds provided $n-c \geq 2$ and $3 \leq c \leq 4$. Moreover if $n-c \geq 1$ and $c>4$, then both Ext-groups above still vanish by [12, and we may in this case replace the assumption of Theorem 3.4 given by the displayed formula with

$$
{ }_{0} \operatorname{Ext}_{D_{3}}^{1}\left(I_{D_{3}} / I_{D_{3}}^{2}, I_{i}\right)=0 \text { for } i=4, \ldots, c-1 \text {. }
$$

Note that in the case $n-c \geq 1$ and $c=2$, the conclusion of Theorem 3.4 holds and, moreover, $\operatorname{Hilb}^{p}\left(\mathbb{P}^{n}\right)$ is smooth at any $(X) \in W(\underline{b} ; \underline{a})$ by [7].

Remark 3.6. For $c>2$ one knows that $\operatorname{Hilb}^{p}\left(\mathbb{P}^{n}\right)$ is not always smooth at any $(X) \in$ $W(\underline{b} ; \underline{a})$ 14. Indeed, since $W(\underline{b} ; \underline{a})$ is irreducible, it is not difficult to find singular points of $\operatorname{Hilb}^{p}\left(\mathbb{P}^{n}\right)$ by first computing its tangent space dimension, $h^{0}\left(\mathcal{N}_{X}\right)$, at a general $(X) \in W(\underline{b} ; \underline{a})$, using Macaulay 2 . Then by experimenting with special choices of $\left(X_{0}\right) \in W(\underline{b} ; \underline{a})$ one may find $h^{0}\left(\mathcal{N}_{X}\right)<h^{0}\left(\mathcal{N}_{X_{0}}\right)$, which means that $\operatorname{Hilb}^{p}\left(\mathbb{P}^{n}\right)$ is singular at $\left(X_{0}\right)$; see [16], which even computes the obstructions of deformations, using Singular, in a related case.

Proof. Due to Theorem 5.1 of [12] we must show that

$$
{ }_{0} \operatorname{Ext}_{D_{i}}^{1}\left(I_{D_{i}} / I_{D_{i}}^{2}, I_{i}\right)=0 \text { for } i=2, \ldots, c-1 .
$$

By assumption we need to prove the vanishing (3.2) for $i=4, \ldots, c-1$ and $c>4$. By induction on $c$ it suffices to show it for $i=c-1, c \geq 5$. Hence it suffices to see that there exist injections

$$
{ }_{0} \operatorname{Ext}_{D_{j+1}}^{1}\left(I_{D_{j+1}} / I_{D_{j+1}}^{2}, I_{c-1}\right) \hookrightarrow{ }_{0} \operatorname{Ext}_{D_{j}}^{1}\left(I_{D_{j}} / I_{D_{j}}^{2}, I_{c-1}\right) \text { for } j=2, \ldots, c-2
$$

By (2.17) and the arguments in the first paragraph of the proof of Proposition 3.1 we may suppose that $\operatorname{depth}_{I\left(Z_{j}\right)} D_{c-1} \geq 3$ for all $j$ satisfying $1<j<c-1$.

We claim that the left-exact sequence (3.1) is also right-exact, i.e. that the rightmost map of the Hom-groups is surjective for $1<j<c-1$. To show this, it certainly suffices to prove $\operatorname{Ext}_{R}^{1}\left(I_{j}, I_{c-1}\right)=0$. However, by paying closer attention to the modules of (3.1), we shall see that also $\operatorname{Ext}_{D_{j}}^{1}\left(I_{j}, I_{c-1}\right)=0$ for $1<j<c-1$ suffices for proving the claim. Indeed if we apply $(-) \otimes_{R} D_{j}$ to $0 \rightarrow I_{D_{j}} \rightarrow I_{D_{j+1}} \rightarrow$ $I_{j} \rightarrow 0$ we get the right-exact sequence $I_{D_{j}} \otimes_{R} D_{j} \rightarrow I_{D_{j+1}} \otimes_{R} D_{j} \rightarrow I_{j} \rightarrow 0$, where $I_{D_{j+1}} \otimes_{R} D_{j} \cong I_{D_{j+1}} / I_{D_{j}} \cdot I_{D_{j+1}}$; and since $I_{D_{j}} \otimes_{R} D_{j+1} \cong I_{D_{j}} / I_{D_{j}} \cdot I_{D_{j+1}}=$ $\operatorname{ker}\left(I_{D_{j+1}} / I_{D_{j}} \cdot I_{D_{j+1}} \rightarrow I_{j}\right)$ we obtain the exact sequence

$$
0 \longrightarrow I_{D_{j}} \otimes_{R} D_{j+1} \longrightarrow I_{D_{j+1}} \otimes_{R} D_{j} \longrightarrow I_{j} \longrightarrow 0,
$$

to which we apply $\operatorname{Hom}_{D_{j}}\left(-, I_{c-1}\right)$. Then we get exactly (3.1) continued to the right by $\operatorname{Ext}_{D_{j}}^{1}\left(I_{j}, I_{c-1}\right)$. Thus the vanishing of $\operatorname{Ext}_{D_{j}}^{1}\left(I_{j}, I_{c-1}\right)$ implies that (3.1) is right-exact.

To see that $\operatorname{Ext}_{D_{j}}^{1}\left(I_{j}, I_{c-1}\right)=0$ we use the isomorphism

$$
\left.\left.\mathcal{H o m}_{\mathcal{O}_{X_{j}}}\left(\mathcal{I}_{j}, \mathcal{I}_{c-1}\right)(-a)\right|_{U_{j} \cap X_{c-1}} \cong \mathcal{O}_{X_{c-1}}\right|_{U_{j} \cap X_{c-1}}
$$

which we obtained in the proof of Proposition 3.1. By (2.9) it follows that

$$
\operatorname{Ext}_{D_{j}}^{1}\left(I_{j}, I_{c-1}\right) \cong H_{*}^{1}\left(U_{j}, \mathcal{H o m}\left(\mathcal{I}_{j}, \mathcal{I}_{c-1}\right)\right) \cong H_{*}^{1}\left(U_{j}, \mathcal{O}_{X_{c-1}}(a)\right)=0
$$


because we have $\operatorname{depth}_{I\left(Z_{j}\right)} D_{c-1} \geq 3$ and hence $\operatorname{depth}_{I\left(Z_{j}\right)} I_{c-1} \geq 3$ ( $I_{c-1}$ is maximally $\mathrm{CM}$ ). This proves the claim. (3.4)

Now we can rewrite the exact sequence (3.1) as

$$
0 \rightarrow D_{c-1}(a) \rightarrow \operatorname{Hom}_{D_{j+1}}\left(I_{D_{j+1}} / I_{D_{j+1}}^{2}, I_{c-1}\right) \rightarrow \operatorname{Hom}_{D_{j}}\left(I_{D_{j}} / I_{D_{j}}^{2}, I_{c-1}\right) \rightarrow 0 .
$$

Sheafifying, restricting to $U_{j} \cap X_{c-1}$ (note that $\mathcal{I}_{D_{j+1}}$ is also locally free on $U_{j} \cap$ $\left.X_{c-1}\right)$ and taking cohomology, we get

$$
\begin{aligned}
\rightarrow H^{1}\left(U_{j}, \mathcal{O}_{X_{c-1}}(a)\right) \rightarrow H^{1}\left(U_{j},\right. & \left.\mathcal{H o m}\left(\mathcal{I}_{X_{j+1}} / \mathcal{I}_{X_{j+1}}^{2}, \mathcal{I}_{c-1}\right)\right) \\
& \rightarrow H^{1}\left(U_{j}, \mathcal{H o m}\left(\mathcal{I}_{X_{j}} / \mathcal{I}_{X_{j}}^{2}, \mathcal{I}_{c-1}\right)\right) \rightarrow
\end{aligned}
$$

Since $\operatorname{depth}_{I\left(Z_{j}\right)} D_{c-1} \geq 3$, the two latter $H^{1}$-groups are by (2.9) isomorphic to the ${ }_{0} \mathrm{Ext}^{1}$-groups quoted in (3.3) and since $H^{1}\left(U_{j}, \mathcal{O}_{X_{c-1}}(a)\right)=0$ we are done.

Remark 3.7. Since (2.17) holds also for $i=3$ provided $\operatorname{dim} D_{c-1} \geq 4, c \geq 5$ and $a_{0}>b_{t}$ by Theorem 2.7, we may continue the proof above to see that the injections (3.3) are isomorphisms for $j \geq 3$. Hence if $X$ is general and $n-c \geq 2$, then

$$
{ }_{0} \operatorname{Ext}_{D_{3}}^{1}\left(I_{D_{3}} / I_{D_{3}}^{2}, I_{c-1}\right) \cong{ }_{0} \operatorname{Ext}_{D_{c-1}}^{1}\left(I_{D_{c-1}} / I_{D_{c-1}}^{2}, I_{c-1}\right) \text {. }
$$

Here the leftmost $\mathrm{Ext}^{1}$-group is computed much faster by Macaulay 2 than the rightmost one. We also get an injection in (3.3) for $j=2$, but now it is not necessarily an isomorphism.

Corollary 3.8. Let $n-c \geq 1, c \geq 5$ and suppose $a_{0}>b_{t}$ and $a_{t+3}>a_{t-1}+$ $a_{t}-b_{1}$. Then $\overline{W(\underline{b} ; \underline{a})}$ is a generically smooth irreducible component of $\operatorname{Hilb}^{p}\left(\mathbb{P}^{n}\right)$ of dimension $\lambda_{c}+K_{3}+\ldots+K_{c}$.

Proof. We get $\operatorname{dim} \overline{W(\underline{b} ; \underline{a})}$ from Theorem 3.2 , Hence by (3.3) and Remark 3.5 it suffices to show that ${ }_{0} \operatorname{Ext}_{R}^{1}\left(I_{D_{2}}, I_{i}\right)=0$ for $4 \leq i \leq c-1$ since ${ }_{0} \operatorname{Ext}_{D_{2}}^{1}\left(I_{D_{2}} / I_{D_{2}}^{2}, I_{i}\right)$ is a subgroup of ${ }_{0} \operatorname{Ext}_{R}^{1}\left(I_{D_{2}}, I_{i}\right)$. First let $i=c-1$. By the Eagon-Northcott resolution (2.3) we see that the largest possible degree of a relation for $I_{D_{2}}$ is $\ell_{2}-b_{1}$ and the smallest possible degree of a generator of $I_{c-1} \cong I_{D_{c}} / I_{D_{c-1}}$ is $\ell_{c}-\sum_{j=t-1}^{t+c-3} a_{j}$. Since $\ell_{c}=\ell_{2}+\sum_{j=t+1}^{t+c-2} a_{j}$, we get ${ }_{0} \operatorname{Ext}_{R}^{1}\left(I_{D_{2}}, I_{c-1}\right)=0$ from

$$
\ell_{2}-b_{1}<\ell_{2}+\sum_{j=t+1}^{t+c-2} a_{j}-\sum_{j=t-1}^{t+c-3} a_{j}=\ell_{2}-a_{t-1}-a_{t}+a_{t+c-2}
$$

i.e. from $a_{t+c-2}>a_{t-1}+a_{t}-b_{1}$. Since we need the vanishing of ${ }_{0} \operatorname{Ext}_{R}^{1}\left(I_{D_{2}}, I_{i}\right)$ for any $i=4,5, \ldots, c-1$, we must suppose $a_{t+3}>a_{t-1}+a_{t}-b_{1}$, and hence we get the corollary.

Remark 3.9. Note that if $c=3$ (resp. $c=4$ ) we can argue as above to see that the conclusions of Corollary 3.8 hold provided $a_{t+1}>a_{t-1}+a_{t}-b_{1}$ (resp. $\left.a_{t+2}>a_{t-1}+a_{t}-b_{1}\right)$. This is, however, proved in [12], Corollary 5.10. For $c \geq 5$, Corollary 3.8 generalizes the corresponding result [12], Corollary 5.9, quite a lot.

\section{Conjectures}

In [10] the first author discovered a counterexample to Conjecture 2.2 for every $c$ in the range $n=c \geq 3$. Indeed the vanishing all $2 \times 2$ minors of a general $2 \times(c+1)$ matrix of linear entries defines a reduced scheme of $c+1$ different points in $\mathbb{P}^{c}$. 
The conjectured dimension of $W(0,0 ; 1,1, \ldots, 1)$ is $c(c+1)+c-2$, while its actual dimension is at most $c(c+1)$.

On the other hand, Theorem 3.2 is quite close to proving Conjecture 2.2 The crucial assumption in Theorem 3.2 is the inequality $a_{t+c-2}>a_{t-2}$ (or $a_{t+3}>a_{t-2}$ if $c>5$ ). Since we, in addition to proving Theorem 3.2, have computed quite a lot of examples where we have $a_{t+c-2}=a_{t-2}$ and $a_{i-\min ([c / 2]+1, t)}>b_{i}$ and each time, except for the counterexample, obtained (2.12) and hence the conjecture, we now want to slightly change Conjecture 2.2 to

Conjecture 4.1. Given integers $a_{0} \leq a_{1} \leq \ldots \leq a_{t+c-2}$ and $b_{1} \leq \ldots \leq b_{t}$, we assume $a_{i-\min ([c / 2]+1, t)} \geq b_{i}$ provided $n>c$ and $a_{i-\min ([c / 2]+1, t)}>b_{i}$ provided $n=c$ for $\min ([c / 2]+1, t) \leq i \leq t$. Except for the family $W(0,0 ; 1,1, \ldots, 1)$ of zero-dimensional schemes above we have, for $W(\underline{b} ; \underline{a}) \neq \emptyset$, that

$$
\operatorname{dim} W(\underline{b} ; \underline{a})=\lambda_{c}+K_{3}+K_{4}+\ldots+K_{c} .
$$

Indeed in the situation of Proposition 2.4 we even expect (2.12) to hold! This will imply Conjecture 4.1 provided the conjecture holds for $W\left(\underline{b} ; \underline{a^{\prime}}\right)$. Note that the conclusion of the conjecture is true provided $n-c \geq 1$ and $2 \leq c \leq 5(\operatorname{char}(k)=0$ if $c=5$ ) by [12.

Finally we will state a conjecture related to the problems (2) and (3) of the Introduction:

Conjecture 4.2. Given integers $a_{0} \leq a_{1} \leq \ldots \leq a_{t+c-2}$ and $b_{1} \leq \ldots \leq b_{t}$, we suppose $n-c \geq 2, c \geq 5$ and $a_{0}>b_{t}$. Then $\overline{W(\underline{b} ; \underline{a})}$ is a generically smooth irreducible component of the Hilbert scheme $\operatorname{Hilb}^{p}\left(\mathbb{P}^{n}\right)$.

Indeed due to the results of this paper and many examples computed by Macaulay 2 in the range $a_{0}>b_{t}$ we even expect the groups ${ }_{0} \operatorname{Ext}_{D_{3}}^{1}\left(I_{D_{3}} / I_{D_{3}}^{2}, I_{i}\right)$ for $i=$ $4, \ldots, c-1$ of Theorem 3.4 to vanish! This will imply Conjecture 4.2. The conclusion of the conjecture may even be true for $0 \leq n-c \leq 1$ (we have no counterexample), but in this range we have verified that the Ext ${ }^{1}$-groups above do not always vanish. Note that the conclusion of Conjecture 4.2 is true provided $n-c \geq 2$ and $2 \leq c \leq 4$ ([7, [11, [12]).

\section{REFERENCES}

[1] A. Beauville, Determinantal hypersurfaces, Michigan Math. J. 48, no. 1 (2000), 39-64. MR.1786479 (2002b:14060)

[2] W. Bruns and J. Herzog, Cohen-Macaulay rings, Cambridge Studies in Advanced Mathematics, 39. Cambridge University Press, Cambridge, 1993. MR1251956 (95h:13020)

[3] W. Bruns and U. Vetter, Determinantal rings, Lecture Notes in Mathematics, 1327, Springer-Verlag, New York/Berlin, 1988. MR953963 (89i:13001)

[4] D.A. Buchsbaum and D. Eisenbud, What annihilates a module? J. Algebra 47 (1977), 231243. MR0476736 (57:16293)

[5] M.C. Chang, A filtered Bertini-type theorem, Crelle J. 397 (1989), 214-219. MR993224 (90i:14054)

[6] D. Eisenbud, Commutative Algebra. With a view toward algebraic geometry, Graduate Texts in Mathematics, 150, Springer-Verlag, 1995. MR1322960 (97a:13001)

[7] G. Ellingsrud, Sur le schéma de Hilbert des variétés de codimension 2 dans $\mathbb{P}^{e}$ a cône de Cohen-Macaulay, Ann. Scient. Éc. Norm. Sup. 8 (1975), 423-432. MR0393020 (52:13831)

[8] D. Grayson and M. Stillman. Macaulay 2-a software system for algebraic geometry and commutative algebra, available at http://www.math.uiuc.edu/Macaulay2/ .

[9] A. Grothendieck, Cohomologie locale des faisceaux cohérents et théorèmes de Lefschetz locaux et globaux, North-Holland, Amsterdam, 1968. MR0476737 (57:16294) 
[10] J.O. Kleppe, Families of low dimensional determinantal schemes. J. Pure Appl. Alg., online 9.11.2010, DOI: 101016/j.jpaa.2010.10.007.

[11] J.O. Kleppe, J. Migliore, R.M. Miró-Roig, U. Nagel and C. Peterson, Gorenstein liaison, complete intersection liaison invariants and unobstructedness, Memoirs Amer. Math. Soc. 154, no. 732, 2001. MR1848976 (2002e:14083)

[12] J.O. Kleppe and R.M. Miró-Roig, Dimension of families of determinantal schemes, Trans. Amer. Math. Soc. 357 (2005), 2871-2907. MR2139931 (2006b:14086)

[13] J. Kreuzer, J. Migliore, U. Nagel and C. Peterson, Determinantal schemes and BuchsbaumRim sheaves, J. Pure Appl. Algebra 150 (2000), 155-174. MR1765869 (2001f:14092)

[14] M. Martin-Deschamps and R. Piene, Arithmetically Cohen-Macaulay curves in $\mathbb{P}^{4}$ of degree 4 and genus 0, Manuscripta Math. 93 (1997), 391-408. MR1457737 (99b:14001)

[15] R.M. Miró-Roig, Determinantal ideals. Progress in Mathematics, 264, Birkhäuser Verlag, Basel, 2008. MR2375719 (2008k:14098)

[16] A. Siqveland, Generalized matric Massey products for graded modules. J. Gen. Lie Theory Appl., in press.

[17] B. Ulrich, Ring of invariants and linkage of determinantal ideals, Math. Ann. 274 (1986), 1-17. MR834101 (87d:14043)

Faculty of Engineering, Oslo University College, Pb. 4 St. Olavs Plass, N-0130 Oslo, NORWAY

E-mail address: JanOddvar.Kleppe@iu.hio.no

Departament d'Algebra i Geometria, Facultat de Matemàtiques, Universitat Barcelona, Gran Via de les Corts Catalanes 585, 08007 Barcelona, Spain

E-mail address: miro@ub.edu 